\title{
Une duplication de I'ADN associée à la maladie de Charcot-Marie
}

La maladie de Charcot-Marie est une neuropathie périphérique impliquant à la fois nerfs sensitifs et moteurs. Sa fréquence est de 1 pour 2500. Son type 1 , le plus fréquent, est caractérisé par une atrophie musculaire distale et une vitesse de conduction nerveuse diminuée. Les signes cliniques apparaissent le plus souvent au début de la $2^{e}$ décennie ; la transmission de cette CM1 est dominante autosomique. Des études de liaison génétique dans des familles étendues suggèrent l'existence d'au moins trois loci: le principal, objet de nombreux travaux depuis 1989, est le locus CM1A situé sur le bras court du chromosome 17 en 17p11-p12; un locus CM1B se trouve sur le chromosome 1 en 1q23-25 [1] ; un $3^{\text {e }}$ type, non encore localisé, est indépendant des deux précédents [2].

Aucune anomalie du génome n'était connue dans le CM. Un groupe de six équipes américaines (douze auteurs) a entrepris [3] l'analyse de 7 familles, dont 6 d'origine francoacadienne et une juive ashkenaze, à l'aide de marqueurs couvrant la portion proximale de $17 \mathrm{p} ; 7$ polymorphismes informatifs montraient des lod scores excédant 3 , les plus élevés atteignant 16 , confirmant une liaison étroite entre la maladie et le bras court du 17 dans ces familles. Deux marqueurs ne montraient aucun recombinant, permettant de cibler l'attaque du locus CM1. Des précisions supplémentaires furent ainsi obtenues avec des marqueurs localisés au locus D17S122, étroitement lié à CM1A [4]. Les sondes utilisées sont des répétitions $\mathrm{GT}(\mathrm{n})$ hautement polymorphiques; avec ces sondes les sujets normaux étaient homo- ou hétérozygotes pour les deux allèles $\mathrm{GT}(\mathrm{n})$. Or 6 des 8 malades étudiés présentaient trois allèles. Un examen portaient que deux allèles, révéla que l'un de ces deux était présent en deux exemplaires : tous les malades possédaient donc trois copies du locus D17S122, qui a ainsi subi une duplication dans la CM1A. Cette duplication a été retrouvée dans les 63 méioses informatives provenant de malades, et chez aucun sujet normal, membre non atteint des familles à risque ou témoins (lod score 19). Les mêmes résultats furent obtenus avec d'autres sondes informatives au même locus, les sujets affectés transmettant à ceux de leurs descendants qui le sont aussi une dose double d'ADN de ce locus. Une tentative de localisation plus précise sur le chromosome fut faite en se servant des références du CEPH. Une analyse de liaison multipoint permit de définir un intervalle de $3 \mathrm{cM}$ incluant la sonde $G T(n)$.

Une forme clinique sévère avait été rapportée antérieurement chez un enfant dont les deux parents étaient atteints et une homozygotie suspectée mais non prouvée [5]. Un cas de même type existait dans une des familles incluses dans le travail de Lupski et al. [3]. Des deux enfants issus de ce mariage entre deux sujets malades, l'un avait une forme classique alors que la maladie de l'autre avait débuté dès la première année et que la vitesse de conduction nerveuse était particulièrement basse. Pour démontrer l'homozygotie, les deux chromosomes 17 de la mère et de chaque enfant ont fait l'objet de préparations séparées d'hybrides somatiques. Chaque chromosome fut ensuite analysé par la sonde GT(n). Chez la mère et l'enfant atteint de la forme habituelle, un seul des allèles portait une duplication; dans la forme grave les deux allèles en étaient porteurs, signant ainsi l'homozygotie. La maladie CM1A, dans laquelle l'homozygote est plus gravement affecté que l'hétérozygote, peut-être appelée "semidominante ", comme c'est le cas par exemple dans la maladie de Willebrandt, et contrairement à celui de la maladie de Huntington, maladie "dominante vraie ", puisque les homozygotes ne sont pas plus atteints que les hétérozygotes.

Enfin une tentative fut faite d'évaluer la taille de la zone dupliquée à l'aide de l'électrophorèse en champ pulsé après action d'enzymes de restriction. L'enzyme SacII formait deux fragments de 600 et 550 pb. Chez les malades, de souche acadienne comme ashkenaze, un fragment supplémentaire de $500 \mathrm{pb}$, héritable selon le mode mendélien, fut mis en évidence.

Les auteurs [3] ont donc démontré qu'une " trisomie fragmentaire " est à l'origine de la CM1A chez des sujets de deux origines ethniques différentes. Ils proposent un procédé de diagnostic de CM1A en utilisant la sonde $G T(n)$. Ils suggèrent qu'un même mécanisme pourrait être à l'œuvre dans d'autres maladies génétiques. Un certain nombre de questions restent soulevées, pour lesquelles n'existent que des hypothèses non encore étayées : par quel mécanisme se produit la duplication ? comment provoque-t-elle la maladie, si elle est réellement causale ? sa taille permet d'envisager un effet de dose si des gènes y sont présents, ou un effet de position. Une analyse du segment dupliqué, notamment des points de jonction, apportera sans doute des éclaircissements.

Un dernier point d'intérêt est l'existence possible de modèles animaux : les candidats sont les mutants de souris trembler ( $\mathrm{Tr}$, dominant) et trembler- $J$ ( $\mathrm{Tr}$ ', semidominant) atteints d'une neuropathie démyélinisante avec con- 
duction nerveuse ralentie. Le locus est situé sur le chromosome 11, synténique du chromosome 17 humain. Il ne s'écoulera sans doute pas un long temps avant la démonstration qu'une duplication existe ou non dans le chromosome 11 de ces animaux. En outre, des projets semblent exister pour rechercher les effets de la duplication de la portion intéressée du chromosome 17 humain chez des souris transgéniques.

J.C.D.

1. Bird TD, Ott J, Giblett ER. Evidence for linkage of Charcot-Marie-Tooth neuropathy to the Duffy locus on chromosome 1. Am J Hum Genet 1982； 34 : 388-94

2. Chance PF, Bird TD, O'Connell P, et al. Genetic linkage and heterogeneity in type 1 Charcot-Marie-Tooth disease (hereditary motor and sensory neuropathy type 1). Am J Hum Genet 1990 ; 47 : 915-25.

3. Lupski JR, de Oca-Luna RM, Slaugenhaupt $\mathrm{S}$, et al. DNA duplication associated with Charcot-Marie-Tooth disease type 1. Cell $1991 ; 66: 219-32$

4. Vance JM, Barker D, Yamaoka LH, et al. Localization of Charcot-Marie-Tooth disease type la CMT1a) to chromosome $17 \mathrm{p} 11.2$. Genomics 1991; 9 : 623-8.

5. Killian JM, Kloepfer HW. Homozygous expression of a dominant gene for CharcotMarie-Tooth neuropathy. Ann Neurol 1979 ; 5 : 515-22.

$\mathrm{m} / \mathrm{s} n^{\circ} 8$, vol. 7, oclobre 91

口n BRÈVES 口n

- Hérédité mitochondriale paternelle. S'il est une notion largement admise et bien classique, c'est celle de l'hérédité uniquement maternelle du génome mitochondrial chez les mammiferes $\left(m / s \quad n^{\circ} 2\right.$, vol. 7, p. 172). Les ovocytes contiennent, en effet, au moins 100000 copies d'ADN mitochondrial contre 50 à 100 copies pour les spermatozoïdes. De plus, selon les espèces, soit les mitochondries mâles ne pénètrent pas dans l'ovocyte, soit elles y seraient dégradées. En revanche, des arguments parfaitement convaincants d'une hérédité mitochondriale paternelle ont été apportés chez la drosophile [1] et la moule [2]. Des équipes suédoises (Uppsala) et américaines, de New York et Berkeley (CA), viennent maintenant de montrer, en utilisant la PCR, que la transmission paternelle du génome mitochondrial était possible chez la souris, quoique dix mille fois moins fréquente que la transmission du génome maternel [3]. La seule réserve possible qu'inspire ce résultat est que, pour des raisons techniques (il fallait que les $\mathrm{ADN}$ paternels et maternels fussent suffisamment différents pour pouvoir être amplifiés spécifiquement par PCR), ils aient été obtenus grâce à des croisements interspécifiques entre Mus domesticus et Mus spretus. Il est, par conséquent, formellement possible qu'un tel phénomène ne soit pas observé dans des croisements intraspécifiques. On peut imaginer, par exemple, que la dégradation des mitochondries mâles ayant pénétré dans un ovocyte n'intéresserait que celles dont le génome est très proche de celui des mitochondries maternelles, et par conséquent dont les caractéristiques fonctionnelles sont identiques. Les transmissions paternelles chez la drosophile et la moule étaient également interspécifiques, mais peutêtre uniquement parce que des transmissions intraspécifiques seraient beaucoup plus diff́iciles à détecter. $\mathrm{Si}$ ces conclusions sont cependant généralisables, et s'appliquent notamment à l'espèce humaine, elles pourraient amener à une réévaluation de l'ancienneté de l'ancêtre commun à tous les êtres humains vivant à l'heure actuelle, la mère originelle de l'espèce Homo sapiens sapiens... si mère unique il y a, ce qui est discuté. En 1987, R. L. Cann et al., du laboratoire de A. C. Wilson à Berkeley (CA, USA), également auteur des travaux récents commentés ici [3], se fondaient sur une transmission uniquement matriarcale de l'ADN mitochondrial pour faire remonter à 200000 ans la vie, en Afrique, de notre ancêtre commune [4]. Ces déductions furent vivement controversées, l'utilisation d'autres " horloges" pouvant aboutir à des conclusions très différentes. De plus, aucun des vestiges humains étudiés en Afrique ou ailleurs ne vient franchement à l'appui de cette thèse. Il n'en reste pas moins que la datation de la divergence entre les différentes populations humaines à l'aide de l'ADN mitochondrial reste une donnée extrêmement importante... qui doit néanmoins être réexaminée à la lumière de la possibilité d'une transmission paternelle. Cette datation postule, en effet, que la seule source de diversification de l'ADN mitochondrial est son potentiel de mutation. S'il faut y ajouter de possibles recombinaisons entre les génomes paternels et maternels, cela amène à diminuer significativement l'ancienneté de notre ancêtre, qui pourrait être ainsi bien plus proche de nous que nous ne l'imaginions.

[1. Kondo R, et al. Genetics 1990 ; $126: 657-63$.]

[2. Hoeh WR, et al. Science 1991 ; 251: 1488-90.]

[3. Gyllesten U, et al. Nature 1991 352 : 255-7.]

[4. Cann RL, et al. Nature 1987 ; $325: 31-6$.
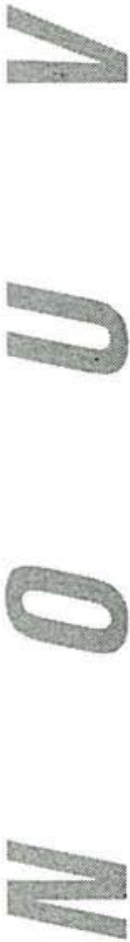